\title{
Ischemic stroke and intracerebral hemorrhage in patients with COVID-19
}

\author{
Hamid Behzadnia', Seyed Nasir Omrani', Hadi Nozari-Golsefid', Saeid Moslemi', \\ Babak Alijani', Zoheir Reyhanian', Mehrnaz Kaviani², Tara Heydari³, \\ Sasan Andalib $4,5,6,7,8$ \\ ${ }^{1}$ Department of Neurosurgery, Poursina Hospital, School of Medicine, \\ Guilan University of Medical Sciences, Rasht, Iran \\ ${ }^{2}$ Amirkabir University of Technology, Tehran, Iran \\ ${ }^{3}$ Georgia Institute of Technology, College of Sciences, Atlanta, Georgia, USA \\ ${ }^{4}$ Neuroscience Research Center, Department of Neurosurgery, Poursina Hospital, School of Medicine, \\ Guilan University of Medical Sciences, Rasht, Iran \\ ${ }^{5}$ Department of Nuclear Medicine, Odense University Hospital, Odense, Denmark \\ ${ }^{6}$ Research Unit of Clinical Physiology and Nuclear Medicine, Department of Clinical Research, Faculty \\ of Health Sciences, University of Southern Denmark, Odense, Denmark \\ ${ }^{7}$ BRIDGE-Brain Research-Inter-Disciplinary Guided Excellence, Department of Clinical Research, \\ University of Southern Denmark, Odense, Denmark \\ ${ }^{8}$ Research Unit of Psychiatry, Department of Psychiatry, Psychiatry in the Region of Southern \\ Denmark, University of Southern Denmark, Odense, Denmark
}

\begin{abstract}
Introduction. Patients with coronavirus disease 2019 (COVID-19) can exhibit neurological symptoms and diseases. A few studies have focused on cerebrovascular diseases in patients with COVID-19. In the present study, we assessed medical records of patients with COVID-19 with stroke.

Material and methods. Forty-seven COVID-19 patients with stroke were consecutively selected and reviewed. Medical records of the patients including information on age, gender, severity of pulmonary involvement (intubation or non-intubation) in COVID, presence of $\mathrm{ICH}$ and ischemic stroke, localization of $\mathrm{ICH}$, history of diabetes and hypertension were collected.

Results. Twenty-three COVID-19 patients (49\%) had ICH and 24 COVID-19 patients (51\%) had ischemic stroke. Sixteen COVID-19 patients with ICH (69.6\%) had lobar hemorrhage and 7 COVID-19 patients with ICH (30.4\%) had non-lobar hemorrhage $(p=0.093)$.

Conclusions. In this study, ICH and ischemic stroke were present at roughly the same rate in COVID-19 patients with stroke. Lobar hemorrhage was seen more frequently in COVID-19 patients with ICH. Brain and cerebrovascular imaging can be a helpful component of the work-up in COVID-19 patients.
\end{abstract}

Keywords: COVID-19, SARS-CoV-2, intracerebral hemorrhage, ischemic stroke

\section{INTRODUCTION}

Coronavirus disease 2019, commonly referred to as COVID-19, stems from severe acute respiratory syndrome coronavirus 2 (SARS-CoV-2). The disease was first introduced in December 2019 and spread throughout the world. The most common symptoms of the disease are fever, cough, shortness of breath and headache; however, COVID-19 has a range of neurological symptoms and diseases. For example, Guillain-Barré syndrome (GBS) [1], meningitis/encephalitis [2], seizures [3], acute disseminated encephalomyelitis [4], and leukoencephalopathy [5] have been reported in COVID-19 patients. Loss of smell (anosmia) and taste (ageusia) was also seen in patients with COVID-19 [6]. 
Recent evidence suggests that COVID-19 can also affect the cardiovascular and cerebrovascular systems. One study reports a Chinese patient with COVID-19 with left hemiparesis due to acute cerebral infarction and occlusion of large blood vessel [7]. Another study reports a patient with COVID-19 with massive intracerebral hemorrhage (ICH) [8]. Cerebrovascular disease was reported in $1.4 \%, 2.8 \%$, and $5.1 \%$ of patients with COVID-19 by Guan et al. [9], Mao et al. [10], and Wang et al., [11], respectively. When comparing severe and moderate COVID-19 patients, acute cerebrovascular diseases $(5.7 \%$ vs. $0.8 \%$ ) was observed more often among severe COVID-19 patients [10]. Helms et al. [12] reported ischemic strokes in 3 of the 13 (23\%) patients. In the present study, we reviewed patients with COVID-19 with cerebrovascular disease.

\section{MATERIALS AND METHOD}

The present retrospective study was carried out in Poursina Hospital of Rasht, Iran. Forty-seven COVID-19 patients with cerebrovascular disease were consecutively selected and reviewed. COVID-19 and cerebrovascular disease were confirmed by lung and head computed tomography (CT). Medical records of the patients including information on age, gender, severity of pulmonary involvement (intubation or non-intubation) in COVID, presence of ICH and ischemic stroke, localization of $\mathrm{ICH}$, history of diabetes and hypertension were collected. Age was also dichotomized in ICH patients as $<50$ years and $\leq 50$ years as ICH is more common in individuals with more than 55 years of age [13]. The study was analyzed by SPSS (version 24) using independent t-test and chi-square analysis. The study was approved by Guilan University of Medical Sciences ethics committee.

\section{RESULTS}

In this study, 47 patients with COVID-19 and stroke were reviewed. Twenty-three patients (49\%) had ICH and 24 patients (51\%) had ischemic stroke (Figure 2). The mean \pm standard deviation (SD) of the age of COVID-19 patients with ICH was $69.43 \pm 9.83$ years, and the mean \pm SD of the age of COVID-19 patients with ischemic stroke was $73.13 \pm 11.76$ years $(\mathrm{P}$-value $>0.05)$.

Of COVID-19 patients with ICH, 12 patients $(52.2 \%)$ were female and 11 patients $(47.8 \%)$ were male $(p=0.99)$. Among COVID-19 patients with ischemic stroke, 11 patients $(45.8 \%)$ were female and 13 patients $(54.2 \%)$ were male $(\mathrm{P}=0.839)$. Out of the 23 COVID-19 patients with ICH, one $(4.3 \%)$ was below the age of 55, and 22 patients $(95.7 \%)$ were over 55 years of age $(\mathrm{p}=0.0001)$. Out of the 24 patients with ischemic stroke, 2 patients $(8.3 \%)$ were below the age of 55, and 22 patients $(91.7 \%)$ were over 55 years of age $(\mathrm{p}=0.0001)$. Sixteen COVID-19 patients with ICH (69.6\%) had lobar hemorrhage (Figure 1) and 7 COVID-19 patients with ICH (30.4\%) had non-lobar hemorrhage $(p=0.093)$. The brain areas affected in all patients with ischemic infarction consisted of middle cerebral artery (MCA) pathways (Figure 2).

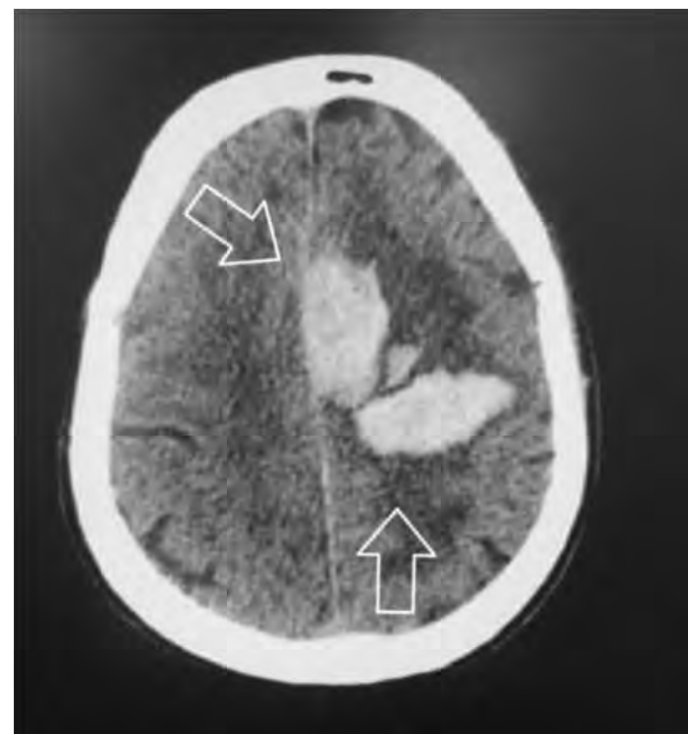

FIGURE 1. Lobar hemorrhage in a patient with COVID-19

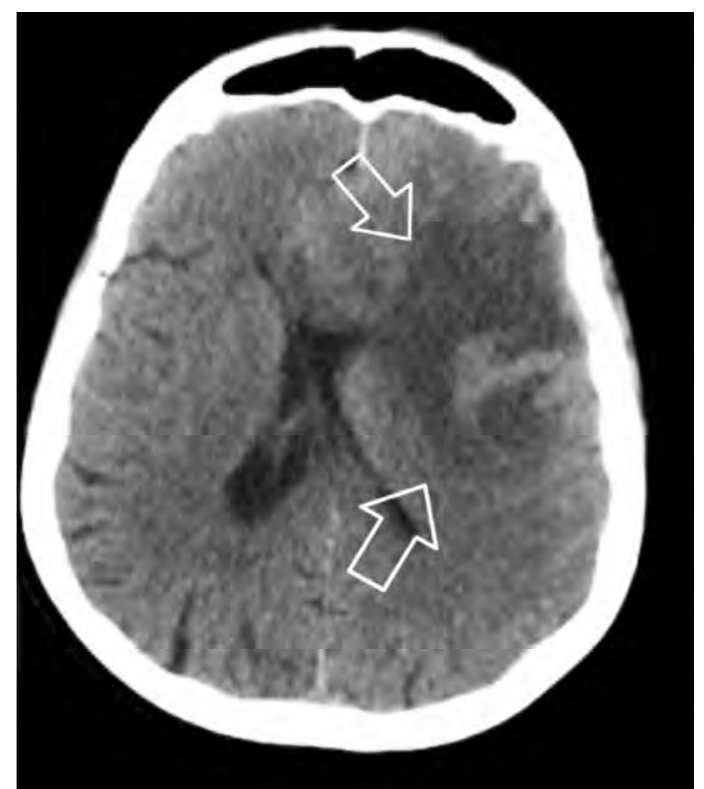

FIGURE 2. Ischemic infarction in middle cerebral artery pathways in a patient with COVID-19 
Eight COVID-19 patients with ICH (34.8\%) were intubated and 15 COVID-19 patients with ICH $(65.2 \%)$ were non-intubated $(\mathrm{P}=0.21)$. There were 18 COVID-19 patients with ischemic stroke (75\%) were intubated and 6 COVID-19 patients with ischemic stroke $(25 \%)$ were non-intubated $(\mathrm{P}=0.023)$.

Two COVID-19 patients with ICH (8.7\%) had a medical history of diabetes mellitus type 2 (DM2); whereas, 5 patients COVID-19 patients with ischemic stroke (20.8\%) had a medical history of diabetes $(p=0.435)$. A medical history of hypertension was found in 8 COVID-19 patients with ICH (34.8\%) and 15 COVID-19 patients with ischemic stroke $(62.5 \%)(\mathrm{P}=0.157)$. Table 1 summarizes demographic information in COVID-19 patients with stroke.

\section{DISCUSSION}

COVID-19 primarily affects the respiratory system, yet it has also shown multi-organ dysfunction. Several lines of evidence have indicated neurotropism of SARS-CoV-2. The SARS-CoV-2 ribonucleic acid (RNA) has been detected in cerebrospinal fluid (CSF) [2]. A wide variety of neurological symptoms and disorders have been reported in patients with COVID-19. It has been demonstrated that SARS-CoV-2 can influence the cerebrovascular system and give rise to stroke. Stroke is a leading cause of death [14] and has serious worldwide health consequences [15], especially in the elderly [16]. Blood pressure (BP) control and anticoagulant administration are implemented to prevent stroke [17]. COVID-19-associated coagulopathy $(\mathrm{CAC})$ is a severe coagulopathy due to inflammation, including inflammatory cytokine storm [18]. Systemic inflammation and inflammatory biomarkers, such as serum IL-6 [19], are associated with risk of ischemic stroke.
Immune thrombocytopenia purpura in a man with COVID-19 with subarachnoid hemorrhage in the right frontal lobe of the brain was reported [20]. An elderly patient with COVID-19 was shown to have a massive ICH in the right hemisphere, in addition to intraventricular and subarachnoid hemorrhage [8]. Five COVID-19 patients (age $<50$ years) with large-vessel stroke were reported [21]. A case report showed a COVID-19 patient with hyperdensity in the superior sagittal, right transverse, and sigmoid sinuses, as well as upper right internal jugular vein indicating thrombosis of cerebral venous sinus [22]. Due to large blood vessel occlusion and acute cerebral infarction, a COVID-19 patient exhibited left hemiparesis [7]. Several arterial thromboses of brain, hands, and legs were demonstrated to be associated with antiphospholipid antibodies in 3 patients with COVID-19 [23].

In $1.4 \%$ of patients with COVID-19 in China, cerebrovascular disease was observed [9]. Mao et al. showed that acute cerebrovascular disease was present in $6(2.8 \%)$ of 214 patients with COVID-19 in Wuhan in China [10]. Compared with severe COVID-19 patients (5.7\%), a low percentage of moderate COVID-19 subjects displayed acute cerebrovascular disease $(0.8 \%)$ [10]. Wang et al. pointed out that cerebrovascular disease was seen in $5.1 \%$ of COVID-19 patients in Wuhan [11]. Ischemic stroke was seen in $3(23 \%)$ out of the 13 patients with COVID-19 in France [12]. In Germany, 3 of 184 patients with COVID-19 suffered from ischemic stroke [24].

Despite relative variability, ICH encompasses $10-20 \%$ of all strokes in most populations [25]. Interestingly, in our study, ICH was seen in $49 \%$ of stroke patients with COVID-19, approximately as much as the rate of ischemic stroke (51\%). To the best of our knowledge, this is the first study reporting an almost equal rate of $\mathrm{ICH}$ and ischemic stroke

TABLE 1. Demographic information in COVID-19 patients with stroke

\begin{tabular}{|c|c|c|c|c|c|c|c|c|c|}
\hline & \multicolumn{2}{|c|}{ Gender } & \multirow{2}{*}{ Age (mean $\pm S D$ ) } & \multirow{2}{*}{ Intubated } & \multirow{2}{*}{$\begin{array}{c}\text { Non- } \\
\text { intubated }\end{array}$} & \multirow{2}{*}{ DM2 } & \multirow{2}{*}{ HTN } & \multicolumn{2}{|c|}{ Age } \\
\hline & Male & Female & & & & & & $<55 y$ & $>55 y$ \\
\hline $\begin{array}{l}\text { Patient with } \\
\text { stroke }\end{array}$ & 24 & 23 & $71.32 \pm 10.97$ & 26 & 21 & 7 & 23 & 3 & 44 \\
\hline Patient with ICH & 11 & 12 & $69.43 \pm 9.83$ & 8 & 15 & 2 & 8 & 1 & 22 \\
\hline $\begin{array}{l}\text { Patient with } \\
\text { ischemic stroke }\end{array}$ & 13 & 11 & $73.13 \pm 11.76$ & 18 & 6 & 5 & 15 & 2 & 22 \\
\hline
\end{tabular}


in patients with COVID-19. The reason why the rate of ICH constitutes a considerable proportion of stroke patients with COVID-19 has not yet been studied. However, a possible mechanism may be increased levels of oxidative stress and the inflammatory response in COVID-19. Divani et al. argued that higher oxidative stress and the inflammatory response may be due to overactivation of the classical pathway of renin angiotensin system (RAS) pathway along with underactivation of the alternative RAS pathway in COVID-19 [18]. SARSCOV-2 binds to the ACE2 receptor on the cellular membrane, which has an important role in RAS [18].

In terms of localization of $\mathrm{ICH}$, non-lobar hemorrhage is more common than lobar hemorrhage in the general population [26]. Interestingly, however, we found that lobar hemorrhage $(69.6 \%)$ is more common than non-lobar hemorrhage $(30.4 \%)$ in ICH patients with COVID-19. It is worth mentioning that downregulation of ACE2, which is presumed to happen by SARS-COV-2, can trigger vasoconstriction through activation of the RAS classical pathway.

In our study and concordant with the findings of Giroud et. al in a general population, we found that most of the ICH patients with COVID-19 were over the age of 55 [13].

Large vessel blockage in ischemic stroke is mostly seen in the territory of the internal carotid artery and the MCA $[27,28]$. In the present study, most of the blockage occurred in the MCA territory in COVID-19 patients with ischemic stroke.

In our study, 34.8\% COVID-19 patients with ICH were intubated; whereas, $75 \%$ of COVID-19 patients with ischemic stroke were intubated.

We found that $8.7 \%$ of COVID-19 patients with ICH had a medical history of diabetes DM2; whereas, 20.8\% COVID-19 patients with ischemic stroke had a medical history of DM2. A medical history of hypertension was found in $34.8 \%$ of COVID-19 patients with ICH and $62.5 \%$ of COVID-19 patients with ischemic stroke.

\section{CONCLUSIONS}

$\mathrm{ICH}$ and ischemic stroke were present at roughly the same rate in COVID-19 patients with stroke. Lobar hemorrhage was seen more frequently in COVID-19 patients with ICH. Brain and cerebrovascular imaging can be a helpful component of the work-up in COVID-19 patients.

Conflict of interest: none declared Financial support: none declared

\section{REFERENCES}

1. Coen M, Jeanson G, Culebras Almeida LA, Hübers A, Stierlin F, Najjar I et al. Guillain-Barré syndrome as a complication of SARS-CoV-2 infection. Brain Behav Immun. 2020:S08891591(20)30698-X.

2. Moriguchi T, Harii N, Goto J, Harada D, Sugawara H, Takamino J et al. A first case of meningitis/encephalitis associated with SARS-Coronavirus-2. Int J Infect Dis. 2020;94:55-8.

3. Karimi N, Sharifi Razavi A, Rouhani N. Frequent Convulsive Seizures in an Adult Patient with COVID-19: A Case Report. Iran Red Crescent Med J. 2020;22(3):e102828.

4. Zhang T, Rodricks MB, Hirsh E. COVID-19-Associated Acute Disseminated Encephalomyelitis: A Case Report. medRxiv. 2020:2020.04.16.20068148.

5. Sachs JR, Gibbs KW, Swor DE, Sweeney AP, Williams DW, Burdette $\mathrm{JH}$ et al. COVID-19-Associated Leukoencephalopathy. Radiology. 2020:201753.

6. Gautier J-F, Ravussin Y. A New Symptom of COVID-19: Loss of Taste and Smell. Obesity. 2020;28(5):848.

7. Zhao J, Rudd A, Liu R. Challenges and Potential Solutions of Stroke Care During the Coronavirus Disease 2019 (COVID-19) Outbreak. Stroke. 2020;51:1356-7.

8. Sharifi-Razavi A, Karimi N, Rouhani N. COVID 19 and Intra cerebral hemorrhage: Causative or Coincidental. New Microbes New Infect. 2020;35:100669.

9. Guan WJ, Ni ZY, Hu Y, Liang WH, Ou CQ, He JX et al. Clinical Characteristics of Coronavirus Disease 2019 in China. N Engl J Med. 2020:[ahead of print]. doi:10.1056/NEJMoa2002032.
10. Mao L, Jin H, Wang M, Hu Y, Chen S, He Q et al. Neurologic Manifestations of Hospitalized Patients With Coronavirus Disease 2019 in Wuhan, China. JAMA Neurol. 2020 [ahead of print].

11. Wang D, Hu B, Hu C, Zhu F, Liu X, Zhang J et al. Clinical Characteristics of 138 Hospitalized Patients With 2019 Novel Coronavirus-Infected Pneumonia in Wuhan, China. JAMA. 2020;323(11):1061-9.

12. Helms J, Kremer S, Merdji H, Clere-Jehl R, Schenck M, Kummerlen $\mathrm{C}$ et al. Neurologic Features in Severe SARS-CoV-2 Infection. N Eng J Med. 2020 [ahead of print].

13. Giroud M, Gras P, Chadan N, Beuriat P, Milan C, Arveux P et al. Cerebral haemorrhage in a French prospective population study. J Neurol Neurosurg Psychiatry. 1991;54(7):595-8.

14. Moadabi Y, Rezaei M, Homaei-Rad E, Fllah Arzpeyma S, Noroozi Guilandehi S, Andalib S. Pineal gland calcification confirmed by CT scan is associated with ischemic stroke. Romanian Journal of Neurology. 2019;18(3):117-20.

15. Andalib S, Divani AA, Michel TM, Høilund-Carlsen PF, Vafaee MS, Gjedde A. Pandora's Box: mitochondrial defects in ischaemic heart disease and stroke. Expert reviews in molecular medicine. 2017;19:e5.

16. Talebi M, Ghertasi M, Taheraghdam A, Andalib S, Sharifipour E. A comparison of risk factors and severity of ischemic stroke in female and male genders in North-West Iran: a cross-sectional study. Iranian Journal of Neurology. 2014;13(4):215.

17. Balaghi-Inalou M, Parsa SA, Gachkar L, Andalib S. Anticoagulant Therapy in Atrial Fibrillation for Stroke Prevention: Assessment of Agreement Between Clinicians' Decision and CHA2DS2-VASc and 
HAS-BLED Scores. High Blood Pressure \& Cardiovascular Prevention. 2018;25(1):61-4.

18. Divani AA, Andalib S, Di Napoli M, Lattanzi S, Hussain MS, Biller J et al. Coronavirus Disease 2019 and Stroke: Clinical Manifestations and Pathophysiological Insights. J Stroke Cerebrovasc Dis. 2020;29(8):104941.

19. Shaafi S, Sharifipour E, Rahmanifar R, Hejazi S, Andalib S, Nikanfar $M$ et al. Interleukin-6, a reliable prognostic factor for ischemic stroke. Iran J Neurol. 2014;13(2):70-6.

20. Zulfiqar A-A, Lorenzo-Villalba N, Hassler P, Andrès E. Immune Thrombocytopenic Purpura in a Patient with Covid-19. N Engl J Med. 2020;382(18):e43-e.

21. Oxley TJ, Mocco J, Majidi S, Kellner CP, Shoirah H, Singh IP et al. Large-Vessel Stroke as a Presenting Feature of Covid-19 in the Young. N Engl J Med. 2020;382(20):e60.

22. Hughes $C$, Nichols T, Pike M, Subbe C, Elghenzai S. Cerebral Venous Sinus Thrombosis as a Presentation of COVID-19. Eur J Case Rep Intern Med. 2020;7(5):001691.

23. Zhang Y, Xiao M, Zhang S, Xia P, Cao W, Jiang W et al. Coagulopathy and antiphospholipid antibodies in patients with COVID-19. N Eng J Med. 2020;382(17):e38.
24. Klok FA, Kruip M, van der Meer NJM, Arbous MS, Gommers D, Kant $\mathrm{KM}$ et al. Incidence of thrombotic complications in critically ill ICU patients with COVID-19. Thromb Res. 2020;191:145-7.

25. Feigin VL, Lawes CM, Bennett DA, Barker-Collo SL, Parag V. Worldwide stroke incidence and early case fatality reported in 56 population-based studies: a systematic review. Lancet Neurol. 2009;8(4):355-69.

26. Lavados PM, Sacks C, Prina L, Escobar A, Tossi C, Araya F et al. Incidence of lobar and non-lobar spontaneous intracerebral haemorrhage in a predominantly Hispanic-Mestizo population--the PISCIS stroke project: a community-based prospective study in Iquique, Chile. Neuroepidemiology. 2010;34(4):214-21.

27. Smith WS, Lev MH, English JD, Camargo EC, Chou M, Johnston SC et al. Significance of large vessel intracranial occlusion causing acute ischemic stroke and TIA. Stroke. 2009;40(12):3834-40.

28. Beumer D, Mulder MJHL, Saiedie G, Fonville S, van Oostenbrugge $\mathrm{RJ}$, van Zwam WH et al. Occurrence of intracranial large vessel occlusion in consecutive, non-referred patients with acute ischemic stroke. Neurovascular Imaging. 2016;2(1):11. 